\title{
Uredinales sobre Blechnaceae, Thelypteridaceae, Schizaeaceae, Myrtaceae, Oxalidaceae, Rhamnaceae, Rubiaceae, Sapindaceae, Smilacaceae e Vitaceae da Reserva Florestal "Armando de Salles Oliveira”, São Paulo, SP, Brasil
}

\author{
Anibal Alves de Carvalho Júnior ${ }^{1,5}$, Mário Barreto Figueiredo ${ }^{2}$, Edson Luiz Furtado ${ }^{3}$ e \\ Joe Fleetwood Hennen ${ }^{4}$
}

Recebido: 21.12.2006; aceito: 25.09.2007

\begin{abstract}
Uredinales on Blechnaceae, Thelypteridaceae, Schizaeaceae, Myrtaceae, Oxalidaceae, Rhamnaceae, Rubiaceae, Sapindaceae, Smilacaceae e Vitaceae of the Forest Reserve "Armando de Salles Oliveira”, São Paulo, SP, Brazil). The aim of this paper is to report the Uredinales collected on members of Blechnaceae, Thelypteridaceae, Schizaeaceae, Myrtaceae, Oxalidaceae, Rhamnaceae, Rubiaceae, Sapindaceae, Smilacaceae and Vitaceae in the Forest Reserve "Armando de Salles Oliveira" at the campus of São Paulo University, São Paulo, SP, Brazil. The rust fungi collected, described and illustrated, as well as their respectives hosts were: Desmella anemiae Syd. \& P. Syd. on Blechnum occidentale L. (Blechnaceae) and Thelypteris dentata (Forssk.) E. St. John (Thelypteridaceae), Endophyllum circumscriptum (Schwein.) Whetzel \& Olive on Cissus selloana Planch. (Vitaceae), Hemileia vastatrix Berk. \& Broome on Coffea arabica L. (Rubiaceae), Phakopsora colubrinae Viégas on Hovenia dulcis Thunb. (Rhamnaceae), Puccinia arechavaletae Speg. on Serjania caracasana Willd. and Serjania communis Cambess. (Sapindaceae), Puccinia lygodii Arthur on Lygodium volubile Sw. (Schizaeaceae), Puccinia oxalidis Dietel \& Ellis on Oxalis latifolia Kunth (Oxalidaceae), Puccinia psidii G. Winter on Psidium guajava L. and Syzygium jambos (L.) Alston (Myrtaceae) and Sphenospora smilacina Syd. on Smilax quinquenervia Vell. (Smilacaceae). Key words: Atlantic Forest, rust fungi
\end{abstract}

RESUMO - (Uredinales sobre Blechnaceae, Thelypteridaceae, Schizaeaceae, Myrtaceae, Oxalidaceae, Rhamnaceae, Rubiaceae, Sapindaceae, Smilacaceae e Vitaceae da Reserva Florestal “Armando de Salles Oliveira”, São Paulo, SP, Brasil). O objetivo do presente trabalho é apresentar as espécies de Uredinales sobre membros de Blechnaceae, Thelypteridaceae, Schizaeaceae, Myrtaceae, Oxalidaceae, Rhamnaceae, Rubiaceae, Sapindaceae, Smilacaceae e Vitaceae da Reserva Florestal da Cidade Universitária “Armando de Salles Oliveira" São Paulo, SP, Brasil. As espécies descritas e ilustradas bem como seus respectivos hospedeiros são: Desmella anemiae Syd. \& P. Syd. sobre Blechnum occidentale L. (Blechnaceae) e Thelypteris dentata (Forssk.) E. St. John (Thelypteridaceae), Endophyllum circumscriptum (Schwein.) Whetzel \& Olive sobre Cissus selloana Planch. (Vitaceae), Hemileia vastatrix Berk. \& Broome sobre Coffea arabica L. (Rubiaceae), Phakopsora colubrinae Viégas sobre Hovenia dulcis Thunb. (Rhamnaceae), Puccinia arechavaletae Speg. sobre Serjania caracasana (Jacq.) Willd. e Serjania communis Cambess. (Sapindaceae), Puccinia lygodii Arthur sobre Lygodium volubile Sw. (Schizaeaceae), Puccinia oxalidis Dietel \& Ellis sobre Oxalis latifolia Kunth (Oxalidaceae), Puccinia psidii G. Winter sobre Psidium guajava L. e Syzygium jambos (L.) Alston (Myrtaceae) e Sphenospora smilacina Syd. sobre Smilax quinquenervia Vell. (Smilacaceae). Palavras-chave: ferrugens, Mata Atlântica

\section{Introdução}

O presente trabalho tem como objetivo divulgar os resultados obtidos nos levantamentos realizados durante um ano, pelo projeto Micota Uredinológica da Reserva Florestal "Armando de Salles Oliveira", São Paulo, SP, Brasil (Carvalho Júnior 2001). Em trabalhos anteriores, Carvalho Júnior et al. (2002a, b, 2004a, 2006) apresentaram as Uredinales sobre plantas das famílias Amaranthaceae, Asclepiadaceae,

1. Instituto de Pesquisas Jardim Botânico do Rio de Janeiro, Rua Pacheco Leão 915, 22460-030 Rio de Janeiro, RJ, Brasil

2. Instituto Biológico, Centro de Sanidade Vegetal, Av. Conselheiro Rodrigues Alves 1252, 04014-002 São Paulo, SP, Brasil

3. Universidade Estadual Paulista, Faculdade de Ciências Agronômicas, Proteção de Plantas, Caixa Postal 236, 18603-970 Botucatu, SP, Brasil

4. Botanical Research Institute of Texas (BRIT), 509 Pecan Street, 76102-4060, Fort Worth, Texas, EUA

5. Autor para correspondência: anibal@jbrj.gov.br 
Asteraceae, Bignoniaceae, Boraginaceae, Convolvulaceae, Cucurbitaceae, Euphorbiaceae, Fabaceae, Malvaceae, Marantaceae, Moraceae e Poaceae. Esta publicação apresenta as Uredinales sobre as famílias Blechnaceae, Thelypteridaceae, Schizaeaceae, Myrtaceae, Oxalidaceae, Rhamnaceae, Rubiaceae, Sapindaceae, Smilacaceae e Vitaceae. Essas publicações, somadas à de Carvalho Júnior et al. (2004b), apresentaram o registro de 87 espécies de Uredinales para a cidade de São Paulo e constituem uma amostragem significativa dos patógenos nesta região. No Estado de São Paulo estão registradas na bibliografia e em herbários cerca de 390 espécies dos patógenos.

Não se dispõem de publicações abrangentes voltadas para as famílias tratadas no presente artigo. Entretanto, os trabalhos de Jackson (1926, 1931a, b), Arthur (1962), Gallegos \& Cummins (1981a, b), Lindquist (1982) e Hennen et al. (2005) abrangem Uredinales sobre várias famílias botânicas nas Américas e são básicos para os estudos sobre o assunto.

\section{Material e métodos}

Os métodos gerais empregados no trabalho seguiram Carvalho Júnior (2001), Carvalho Júnior et al. (2002a, b) e Cummins \& Hiratsuka (2003).

As espécies identificadas estão apresentadas em ordem alfabética na seguinte seqüência: nomes válidos, sinonímias, tipos, estádios esporíferos encontrados, descrições dos táxons, materiais analisados com espécies hospedeiras parasitadas, comentários, distribuição geográfica e ilustrações.

Os espécimes estão preservados no Herbário do Laboratório de Micologia Fitopatológica do Centro de Sanidade Vegetal do Instituto Biológico de São Paulo (IBI) e algumas no Herbário do Instituto de Pesquisas Jardim Botânico do Rio de Janeiro (RB).

\section{Resultados e Discussão}

Desmella anemiae Syd. \& P. Syd., Ann. Mycol. 16: 241. 1918. Uredo aneimiae Henn., Hedwigia 35: 255. 1896. LECTOTIPO: BRASIL. SANTA CAtARINA: Tubarão, sobre Anemia tomentosa var. fulva (Sav.) T. Moore, X-1886, Ule 515.

Figuras 1-3

Anamorfos:

Caeoma mbatobiensis Speg., An. Soc. Cient. Argent.
17: 96. 1884. Desmella mbatobiense Syd. \& P. Syd., Ann. Mycol. 16: 241. 1918. TIPO: PARAGUAI. Propriedade MBatobi, sobre Pteridis?, VII-1883, B. Balansa 3866.

Caeoma superficiale Speg., An. Soc. Cient. Argent. 17: 96. 1884. Desmella superficialis Syd. \& P. Syd., Ann. Mycol. 16: 242. 1918. Desmella superficialis F. Kern, in Stevenson, Fungi of Puerto Rico, Contrib. Reed Herb. p. 264. 1975. TIPO: PARAGUAI. Próximo de Mbatobi, sobre Blechnum sp., VII-1883, B. Balansa 3882.

Uredo gymnogrammes P. Henn., Hedwigia 34: 337. 1895. Desmella gymnogrammes Syd. \& P. Syd., Ann. Mycol. 16: 242. 1918. TIPO: BRASIL. SANTA CATARInA: Blumenau, sobre Gymnogramna sp., s.d., Alfr. Moeler s.n.

Uredo blechnicola P. Henn., Hedwigia 43: 165. 1904. TIPO: BRASIL. Rio Jura, Juruá-Mirim, sobre Blechnum volubile Klf., VIII-1901, Ule 3141.

Uredo nephrolepidis Dietel, Mem. Soc. Neuchâteloise Sci. Nat. 5: 576. 1913. TIPO: COLÔMBIA. Antioquia: Angelopolis-Guaca, sobre Nephrolepis pendula (Raddi) J. Sm., 12-IX-1910, Mayor 151.

III - Teliossoros hipófilos, pequenos, subepidérmicos, surgindo a partir dos estômatos, amarelo-pálidos, não irrompentes, pulverulentos; paráfises ausentes. Teliósporos bicelulares, com um septo vertical, transversalmente elipsóides, (18-)20(-23) $\mu$ m compr., (21-)25(-28) $\mu \mathrm{m}$ larg.; paredes hialinas ou amarelopálidas, lisas, 1,6-7,2 $\mu \mathrm{m}$ espessa. Esporos sustentados num feixe comum, cada um dos esporos pedicelados, separando-se do feixe central mediante um septo. Esse conjunto de células emerge através de um estômato. Pedicelos decíduos.

II - Urediniossoros hipófilos, inicialmente amarelos tornando-se esbranquiçados tardiamente, surgindo a partir dos estômatos, pequenos; paráfises ausentes. Urediniósporos globóides ou ovóides, pulverulentos, (19-)24(-27) $\mu \mathrm{m}$ diâm.; paredes hialinas, equinuladas, 0,9-2,2 $\mu \mathrm{m}$ espessa; poros germinativos não visíveis, pedicelos decíduos. Assim como nos teliósporos, os esporos são sustentados num feixe comum, cada um dos esporos pedicelado, separando-se do feixe central mediante um septo.

0, I - Não são conhecidos os estádios espermogonial e ecial.

Materiais analisados: sobre Thelypteris dentata (Forssk.) E. St. John (Thelypteridaceae): 19-IX-1997, A.A. Carvalho Júnior 97-189 (IBI17959-II); 
24-X-1997, 97-199 (IBI17969-II); 24-XI-1997, 97-215 (IBI17985, RB382835-II, III); 20-II-1998, 98-053 (IBI18069-II, III); 23-IV-1998, 98-302 (IBI18319-II); 26-V-1998, 98-333 (IBI18350-II); 25-VII-1998, 98-355 (IBI18372-II) e Blechnum occidentale L. (Blechnaceae): 29-VII-1997, A.A. Carvalho Júnior 97-142 (IBI17912, RB382820-II); 18-VIII-1997, 97-168 (IBI17938-II).

Comentários: segundo Hennen et al. (2005) existe apenas uma espécie de Desmella que pode ser encontrada nas regiões neotropicais e que tem como hospedeiras espécies de samambaias. As espécies listadas anteriormente como sinonímias estavam separadas originalmente, devido aos diferentes hospedeiros ou diferenças desprezíveis no tamanho dos esporos anamórficos, espessura das paredes dos esporos, visibilidade dos poros germinativos e equinulação. Devido aos esporos serem produzidos em soros supraestomatais, esporos de diferentes idades podem ser utilizados na preparação de lâminas para microscopia. É difícil determinar se os esporos que estão sendo descritos estão maduros. Nos protólogos das espécies de Desmella colocadas como sinônimos anamórficos no presente artigo não constam descrição de teliósporos. As medições dos teliósporos foram retiradas do material IBI17985 e dos urediniósporos do material IBI17959. A espessura da parede dos teliósporos são bastante irregulares; sendo assim foi considerada, neste artigo, a maior espessura encontrada em cada teliósporo. Apesar dos urediniósporos apresentarem pedicelos os mesmo são decíduos e os hilos não são visíveis, permitindo apenas a medição dos diâmetros.

Distribuição geográfica: Neotropical, do México à Argentina, e mais recentemente no Hawai (Hennen et al. 2005).

Endophyllum circumscriptum (Schwein.) Whetzel \& Olive in Olive \& Whetzel, Am. J. Bot. 4: 49. 1917. LECTÓTIPO: PORTO RICO. MAYAGUEZ: sobre Cissus sicyoides L., 29-III-1916, Whetzel \& Olive 82.

Figuras 4-6

Aecidium guttatum Kunze in Weigelt, Exsicc. Sine no. 1827. Endophyllum guttatum Syd. \& P. Syd., Ann. Mycol 18: 179. 1920. TIPO: SURINAME. s.1., sobre Cyssus sicyoides L. (originalmente como Compositae?) s.d., Weigelt s.n. (in Saccardo, Sylloge Fungorum v. 11, p. 217. 1895).
Aecidium circumscriptum Schwein. in Berkeley \& Curtis, J. Acad. Nat. Sci. Philadelphia 2: 283. 1853. TIPO: SURINAME. s.l., sobre Cissus sp., s.d., Weigelt s.n.

Aecidium cissi Winter, Hedwigia 23: 168. 1884. TIPO:

BRASIL. Santa Catarina: perto de São Francisco, sobre Cissus sicyoides L., III-1884, Ule 51.

III - Teliossoros anfígenos, predominantemente hipófilos, peridiados, amarelos, numerosos, agrupados, algumas vezes em áreas hipertrofiadas e pulvinadas, pequenos, $0,1 \mu \mathrm{m}$ diâm.; perídio recurvado, quebrado em alguns segmentos, células 13-20 $\mu$ m diâm., parede 1-2,6 $\mu \mathrm{m}$ espessa, estriada externamente e verrugosa internamente; paráfises ausentes. Teliósporos catenulados, mais ou menos globóide-angulares ou irregulares devido à pressão, (14-)17(-19) $\mu$ m diâm.; parede hialina a amarelada, densamente verrugosa, fina, 0,7-1,5 $\mu \mathrm{m}$ espessa; poros germinativos não visíveis.

II, 0, I - Estádios ecial e uredinial desconhecidos.

Materiais examinados: sobre Cissus selloana (Baker) Planch. (Vitaceae): 22-XII-1997, A.A. Carvalho Júnior 97-235 (IBI18005-III); 13-I-1998, 98-019 (IBI18036, RB832836-III); 23-I-1998, 98-026 (IBI18043-III); 20-II-1998, 98-035 (IBI18051-III); 31-III-1998，98-280 (IBI18297-III); 23-IV-1998, 98-298 (IBI18315-III); 26-V-1998, 98-321 (IBI18338-III); 25-VII-1998, 98-347 (IBI18364-III).

Comentários: os esporos da espécie-tipo de Aecidium guttatum, A. circunscriptum e A. cissi não foram germinados e isso impossibilitou colocá-los no gênero Endophyllum. Porém, como a descrição morfológica foi feita a partir de teliósporos (Endophyllum circunscriptum é uma espécie microcíclica) e seus esporos são indistintos morfologicamente do gênero Aecidium, neste caso o gênero Aecidium (gênero anamórfico) pode ser considerado um sinônimo teleomórfico. O lectótipo de E. circumscriptum foi designado por Buriticá \& Hennen (1980). Segundo Olive \& Whetzel (1917) o estádio 0 contém espermogônios epífilos, poucos, subepidermais, raramente rompendo a epiderme, com aproximadamente 80-85 $\mu \mathrm{m}$ larg. quando seccionados; porém, no material estudado, não foram observados.

Distribuição geográfica: Sudoeste dos Estados Unidos da América, Ilhas do Caribe, México até a Argentina (Buriticá \& Hennen 1980). 
Hemileia vastatrix Berk. \& Broome, in Berkeley, Gard. Chron. 45: 1157. 1869. TIPO: SRI LANKA. s.l., sobre Coffea sp., V-1869, Thwaites s.n. Figuras 7-9

III - Teliossoros hipófilos, em manchas cloróticas, amarelo-pálidos, surgindo a partir dos estômatos, ou no mesmo ponto dos urediniossoros, pulverulentos, muito dispersos, muito pequenos; paráfises ausentes. Teliósporos em forma de nabo ou globóides, mais ou menos umbonados, (15-)18(-23) um compr., (13-)19(-25) $\mu \mathrm{m}$ larg.; parede amarelo-pálida ou hialina, ligeiramente mais grossa acima, lisa, delgada, $1,3 \mu \mathrm{m}$ espessa; poros germinativos não visíveis; pedicelos decíduos, hialinos, do mesmo comprimento ou da metade do comprimento dos teliósporos, finos. Teliósporos germinam sem dormência.

II - Urediniossoros hipófilos, em manchas cloróticas, laranja-claro-pálidos ou amarelo-pálidos, pulverulentos, surgindo a partir dos estômatos e raramente rompendo a epiderme ao redor dos estômatos, dispersos, ou raramente em grupos formando círculos concêntricos, muito pequenos; paráfises ausentes. Urediniósporos com pedicelos decíduos, pouco obovóides, achatados do lado ventral, (26-)29(-34) $\mu \mathrm{m}$ compr., (16-)19(-22) $\mu \mathrm{m}$ larg.; parede amarelo-pálida, às vezes mais largas e papilosas, com equinulações pontiagudas no lado dorsal, lado ventral liso, 1-3,2 $\mu \mathrm{m}$ espessa na parte lateral; poros germinativos obscuros.

0, I - Estádios espermogonial e ecial desconhecidos.

Material examinado: sobre Coffea arabica L. (Rubiaceae): 29-VII-1997, A.A. Carvalho Júnior 97-139 (IBI17909-II); 19-IX-1997， 97-192 (IBI17962-II); 24-X-1997, 97-197 (IBI17967-II, III); 22-XII-1997, 97-238 (IBI18008-II); 13-I-1998, 98-003 (IBI18020-II); 20-II-1998， 98-048 (IBI18064-II); 31-III-1998, 98-264 (IBI18281-II); 23-IV-1998, 98-292 (IBI18309-II); 26-V-1998, 98-322 (IBI18339-II)，25-VII-1998，98-344 (IBI18361-II).

Comentários: segundo Hennen \& Figueiredo (1984) Hemileia vastatrix poderia apresentar um ciclo de vida bastante curioso sendo autoécia de ciclo longo, sem espermogônios reconhecíveis e com écios morfologicamente semelhantes ou idênticos aos uredínios. Se houvesse a redução endofilóide do ciclo de vida, então os teliósporos seriam semelhantes aos eciósporos, que por sua vez são idênticos aos urediniósporos. Isso explicaria a ocorrência de teliósporos semelhantes a "urediniósporos", referidos em trabalhos provenientes da Índia e Portugal (Rajendren 1967, Rodrigues Júnior et al. 1980). Nestas localidades haveriam formas microcíclicas do tipo "Endophyllum" derivadas da população macrocíclica original.

Distribuição geográfica: América Central, do Sul, no oeste da África, Ásia, Filipinas e Ilhas do Pacífico. Onde quer que o café seja cultivado (Hennen et al. 2005).

Phakopsora colubrinae Viégas, Bragantia 19 (nota 20): CIII. 1960. TIPO: BRASIL. Minas GeraIs: Estação Experimental de Água Limpa, sobre Colubrina rufae Reiss., 23-VI-1946, E.P. Heringer 458.

Figura 10

Anamorfos

Malupa colubrinae (Cummins) Buriticá \& J.F. Hennen, in Buriticá, Rev. Acad. Colomb. Cienc. Exactas Fis. Nat. 23: 296. 1999. Uredo colubrinae Cummins, Bull. Torrey Bot. Club 70: 79. 1943. TIPO: GUATEMALA. QUETZALTENANGO: sobre Colubrina ferruginosa Brongn., 20-II-1941, Standley 87894.

Uredo hoveniae J.C. Lindq. \& Costa Neto, Revista Fac. Agron. Un. Nac. La Plata 43: 61. 1967. TIPO: BRASIL. Rio Grande do Sul: Taquari, sobre Hovenia dulcis Thunb., 9-VII-1948, Costa Neto s.n.

III - Estádio telial não encontrado no material analisado. II - Urediniossoros hipófilos, em grupos, em manchas necróticas bem delimitadas, canela-pálidos, abertos por um poro, pulverulentos, circulares, pequenos, 0,1-0,4 $\mu \mathrm{m}$ diâm.; paráfises cilíndricas a clavadas, quebradiças, 18-60 $\mu \mathrm{m}$ compr., 5-10 $\mu \mathrm{m}$ larg., paredes hialinas, delgadas, de espessura uniforme, ocasionalmente $2 \mu \mathrm{m}$ espessa no ápice. Urediniósporos sésseis, obovóides a elipsóides, (18-)20(-23) $\mu \mathrm{m}$ compr., (13-)15(-17) $\mu \mathrm{m}$ larg.; parede amarelada a canela-pálida, com abundantes equinulações pequenas, $0,7-1,4 \mu \mathrm{m}$ espessa lateral, uniforme; poros germinativos 4-6, quase imperceptíveis, próximos ao equador.

0 , I - Os estádios espermogonial e ecial são desconhecidos.

Materiais examinados: sobre Hovenia dulcis Thunb. (Rhamnaceae): 18-VIII-1997, A.A. Carvalho Júnior 97-158 (IBI17928-II); 24-X-1997, 97-194 (IBI17964-II); 24-XI-1997, 97-213 (IBI17983-II); 22-XII-1997, 

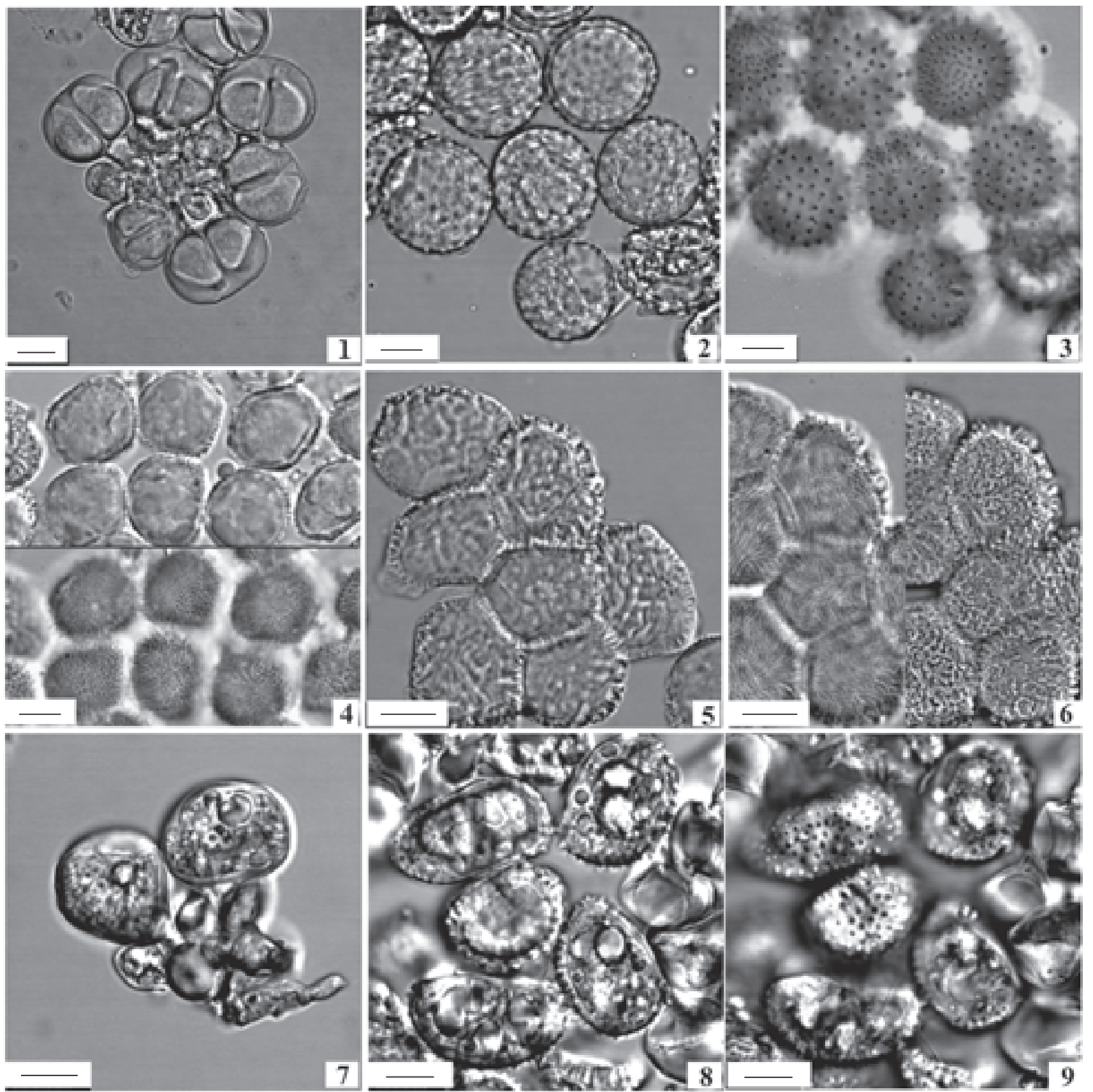

Figuras 1-3. Desmella anemiae (IBI17985). 1. Teliósporos em vários estádios de maturação ainda presos à célula esporogênica. 2-3. Urediniósporos. 2. Em vista mediana. 3. Em vista superficial. Figuras 4-6. Endophyllum circunscriptum (IBI18036). 4. Teliósporos - acima em vista mediana e abaixo em vista superficial. 5-6. Perídio do teliossoro. 5. Em vista mediana. 6. À esquerda em vista superficial externa e à direita em vista superficial interna. Figuras 7-9. Hemileia vastatrix (IBI17967). 7. Teliósporos ainda aderidos à célula esporogênica. 8-9. Urediniósporos. 8. Em vista mediana. 9. Em vista superficial. Barras $=10 \mu \mathrm{m}$. 
97-233 (IBI18003-II); 13-I-1998，98-016 (IBI18033-II); 23-I-1998, 98-027 (IBI18044-II); 20-II-1998, 98-047 (IBI18063-II); 31-III-1998, 98-273 (IBI18290-II); 23-IV-1998， 98-311 (IBI18328-II); 26-V-1998, 98-314 (IBI18331-II); 25-VII-1998, 98-352 (IBI18369, RB382857-II).

Comentários: segundo Buriticá (1999) esta espécie é uma ferrugem neotropical nativa e foi capaz de infectar Hovenia dulcis depois desta hospedeira ter sido introduzida no Brasil proveniente da Ásia. Este autor descreve os télios da seguinte maneira:

III - teliossoros hipófilos, ao redor dos urediniossoros, lenticulares, crescimento determinado, isolados, 5-7 extratos de esporos; himênio subepidermal, suavemente côncavo; teliósporos irregularmente organizados, superiores marron-pálidos, inferiores hialinos, elipsóides, cubóides, 10-18 ×6-10 $\mu \mathrm{m}$; parede hialina nos esporos inferiores e marron-pálida nos esporos superiores, 1-1,5 $\mu \mathrm{m}$ espessa, 2-4 $\mu \mathrm{m}$ no ápice dos teliósporos do extremo superior.

Distribuição geográfica: América Central e do Sul (Buriticá 1999).

Puccinia arechavaletae Speg., An. Soc. Cient. Argent. 12: 67. 1881. TIPO: URUGUAI. Montevideo, sobre Cardiospermum velutinum Hook. \& Arn., VII-1881, Arechevaleta s.n.

Figura 11

Uromyces pervius Speg., An. Soc. Cient. Argent. 17: 94. 1884. TIPO: PARAGUAI. GUAIRÁ: Villa Rica, sobre Cupania sp., I-1882, Balansa 3515.

Uromyces aeruginosus Speg., Rev. Argentina Hist. Nat. 1: 175. 1891. TIPO: PARAGUAI. ITAPÚA: General Delgado, Posca-cue, sobre Sapindaceae indet., IV-1884, Balansa 4267.

Puccinia serjaniae Ellis \& Everhart, Erythea 5: 6. 1897. TIPO: MÉXICO. BAJA CAlifornia: Pescadero, sobre Serjania sp., IX-1893, A.W. Anthony s.n.

Puccinia anguriae Arthur \& Cummins, Ann. Mycol. 31: 43. 1933. TIPO: BRASIL. RIO DE JANEIRO: Cabo Frio, sobre Sapindaceae indet., 8-VIII-1915, Rose \& Russell 20704.

III - Teliossoros hipófilos, em manchas necróticas, castanho-escuros, acinzentados quando germinados, pulvinados, compactos, isolados ou agrupados em círculos concêntricos, circulares, pequenos, 0,1-1 mm diâm.; paráfises ausentes. Teliósporos bicelulares elipsoidais, arredondados em ambos os extremos, pouco ou nada constritos no septo, (20-)26(-29) $\mu \mathrm{m}$ compr., (16-)20(-24) $\mu \mathrm{m}$ larg.; parede castanha, lisa, 1,3-2,7 $\mu \mathrm{m}$ espessa; poros germinativos não visíveis; pedicelo persistente, hialino, grande e flexuoso com até $59 \mu \mathrm{m}$ compr., 6,4 $\mu \mathrm{m}$ larg. próximo ao hilo. Teliósporos bicelulares escassos em relação aos mesósporos globóides, ovóides ou piriformes, (18-)21(-25) $\mu \mathrm{m}$ compr., (16-)19(-24) $\mu \mathrm{m}$ larg., parede castanha, lisa, no entanto ligeiramente mais grossa acima, 1,5-2,6 $\mu \mathrm{m}$ espessa; pedicelos dos mesósporos com até $67 \mu \mathrm{m}$ compr., 5,1 $\mu \mathrm{m}$ larg. próximo ao hilo. Poucos mesósporos apresentam umbo.

0, I, II - Estádios espermogonial, ecial e uredinial não conhecidos.

Materiais examinados: sobre Serjania caracasana (Jacq.) Willd. (Sapindaceae): 24-11-1997, A.A. Carvalho Júnior 97-211 (IBI17981-III); 22-XII-1997, 97-239 (IBI18009-III); 13-I-1998, 98-012 (IBI18029-III); 20-II-1998， 98-044 (IBI18060, RB382839-III); 31-III-1998, 98-279 (IBI18296-III); 23-IV-1998, 98-312 (IBI18329-III); 26-V-1998, 98-331 (IBI18348-III); 25-VII-1998, 98-336 (IBI18353-III) e sobre Serjania communis Cambess. (Sapindaceae): 29-VII-1997, A.A. Carvalho Júnior 97-149 (IBI 17919-III); 24-X-1997, 97-196 (IBI17966, RB382833-III); 24-XI-1997, 97-218 (IBI17988-III).

Comentários: a espécie caracteriza-se pela grande quantidade de mesósporos.

Distribuição geográfica: espécie muito comum sobre Sapindaceae em toda América Central e do Sul (Lindquist 1982).

Puccinia lygodii Arthur, Bull. Torrey Bot. Club. 51: 55. 1924. TIPO: BRASIL. BAHIA: sobre Lygodium polymorphum (Cav.) Kunth, 28-V-1915, Rose \& Russell 19664a.

Figuras 12-13

Anamorfos:

Uredo lygodii Hariot, J. Bot. 14: 117. 1900. TIPO:

BRASIL. Pernambuco: sobre Lygodium sp., 28-V-1915, Gardener 1229.

Milesina lygodii Syd. Mycologia 17: 255. 1925. TIPO:

SURINAMI. POTARO-SIPARUNI: Tumatumari, sobre

Lygodium sp., 11-II-1922, Stevens 54.

III - Estádio telial não encontrado no material examinado.

II - Urediniossoros hipófilos, em áreas necróticas, de coloração canela, dispersos ou algumas vezes agregados, de origem subepidermal, posteriormente 
em parte recoberto pela epiderme, pulverulentos, ruptura da epiderme inconspícua, 0,3-0,5 mm diâm., circulares; paráfises ausentes. Urediniósporos com pedicelos decíduos, obovóides a elipsóides, (20-)26(-30) $\mu \mathrm{m}$ compr., (19-)21(-26) $\mu \mathrm{m}$ larg.; parede amarela, equinulada, 0,9-1,5 $\mu \mathrm{m}$ espessa, geralmente com áreas irregulares lisas (sem equinulações); poros germinativos indistintos, provavelmente 2 ou 3 equatoriais.

0, I - Estádios espermogonial e ecial são desconhecidos.

Materiais analisados: sobre Lygodium volubile Sw. (Schizaeaceae): 23-I-1998, A.A. Carvalho Júnior 98-023 (IBI18040-II); 20-II-1998， 98-051 (IBI18067-II); 31-III-1998, 98-271 (IBI18288-II); 23-IV-1998, 98-284 (IBI18301, RB382843-II); 26-V-1998, 98-332 (IBI18349-II).

Comentários: devido aos soros urediniais serem recobertos pela epiderme delgada, sua observação sob lupa nos dá impressão de que tratam-se de bolhas distribuídas pelo limbo foliar. Segundo Sotão et al. (2001), o estádio III constitui-se de teliossoros hipófilos, dispersos, circularets ou oblongos, 0,5-1 mm, expostos deste cedo, pulverulentos, de coloração canela, ruptura da epiderme inconspícua; esporos pedicelados, globóides, elipsóides ou obovóides, 24-29 $\mu \mathrm{m} \times 19-23 \mu \mathrm{m}$, arredondados no ápice, base arredondada ou estreita em alguns, sem constrição no septo; parede marron-canela-pálida, 1,5 $\mu \mathrm{m}$ espessa, mais grossa no ápice (3-7 $\mu \mathrm{m})$, lisa, septo variavelmente oblíquo; pedicelo hialino, mais ou menos do mesmo comprimento do esporo.

Distribuição geográfica: Luisiana, Flórida (Estados Unidos da América), América Central, Venezuela, Guianas, Colômbia e Brasil (Hennen et al. 2005).

Puccinia oxalidis Dietel \& Ellis in Dietel, Hedwigia 34: 291. 1895. TIPO: MÉXICO. s.1., sobre Oxalis sp., 1895, E. Palmer s.n.

Figura 14

Anamorfo

Uredo oxalidis Lév., Ann. Sci. Nat. II, 16: 240. 1841. Argomyces oxalidis (Lév.) Arthur, N. Am. Flora 7: 217. 1912. Dicaeoma oxalidis (Lév.) Kuntze, Rev. Gen. 3: 469. 1898. TIPO: VENEZUELA. s.l., sobre Oxalis sp., s.d., s.col.

Uredo oxalidearum Cooke, Grevillea 10: 123. 1882. TIPO: VENEZUELA. Caracas, sobre Oxalis corniculata L., s.d., Ernst. s.n.
III, 0, I - Os estádios telial, espermogonial e ecial não foram encontrados nos materiais analisados.

II - Urediniossoros hipófilos, amarelo-pálidos, expostos desde cedo, a princípio ceroso, pulverulento tardiamente, rompendo a epiderme inconspicuamente, algumas vezes confluêntes, em círculos concêntricos ou em grupos irregulares, 0,2-0,4 mm diâm.; paráfises ausentes. Urediniósporos com pedicelos decíduos, globóides ou elíptico-globóides (15-)17(-19) $\mu$ m compr., (13-)14(-15) $\mu \mathrm{m}$ larg.; parede amarela-pálida, pouco equinulada, fina, 1-1,6 $\mu \mathrm{m}$ espessa; poros germinativos obscuros.

Materiais analisados: sobre Oxalis latifolia Kunth. (Oxalidaceae): 24-XI-1997, A.A. Carvalho Júnior 97-217 (IBI 17987-II); 25-VII-1998，98-356 (IBI18373-II).

Comentários: Puccinia oxalidis é muito comum na América tropical e subtropical sobre muitas espécies de Oxalis e foi introduzida na Austrália, Japão e provavelmente em outros lugares (Hennen et al. 2005). Long \& Harsch (1918) relataram que os resultados de inoculações indicaram que o espermogônio e écio de $P$. oxalidis ocorrem em Mahonia repens (Lindl.) G. Don. no Novo México e nos Estados Unidos da América, embora estes resultados nunca foram confirmados por experimentos de inoculações adicionais, nem por observações de campo. Duas outras ferrugens foram descritas como produzindo espermogônios e écios sobre Oxalis spp. no hemisfério ocidental: Puccinia andropogonis Schwein., que produz uredínios e télios sobre Andropogon spp. e Puccinia sorghi Schwein. que produz uredínios e télios em Zea spp.

Arthur (1920) referindo-se a uma revisão da nomenclatura de Puccinia oxalidis para Dicaeoma oxalidis (Lév.) Kuntze, que atualmente é considerada nula, descreve os estádios esporíferos da seguinte maneira:

0-I - sobre Berberidaceae: Mahonia aquifolium (Pursh) Nutt. no Novo México e Mahonia swaseyi (Buckl.) Fedde no México.

0 - espermogônios epífilos, frouxamente gregários, em grupos de 1-3 mm diâm., conspícuos, profundos, marron-chocolate, oblongo-elipsóides, 185-225 $\mu \mathrm{m}$ compr., 110-125 $\mu \mathrm{m}$ larg.; filamentos curtos ostiolares não projetados além do ostíolo.

I - écios hipófilos, agrupados em áreas com 3-7 mm diâm., em forma de chifre, estreito acima e agudo no 
ápice, 0,2 mm compr.; perídio branco, fechado e também irrompendo o tecido do hospedeiro; células peridiais quadradas ou rômbicas, $20-26 \times 18-23 \mu \mathrm{m}$; parede exterior 9-12 $\mu \mathrm{m}$ espessa, transversalmente estriada; parede interior 1,5-3 $\mu \mathrm{m}$, proximamente e finamente verrugosa, aparentando ser lisa quando úmida.

III - télios hipófilos, em grupos circulares ou em grupos irregulares, 2-5 mm diâm., cobrindo toda a superfície, circulares, 0,3 mm diâm., algo confluêntes, desde cedo expostos, marrom-amarelados muito pálidos, cerosos, irrompentes, inconspícuos; teliósporos elipsóides ou largamente oblongos, 18-28 $\times 13-20 \mu \mathrm{m}$, arredondados ou obtusos em ambos os extremos, sem constrição ou pouco constritos no septo; parede hialina, lisa, uniformemente fina $0,5-0,8 \mu \mathrm{m}$; pedicelos hialinos, grossos e muito curtos.

Distribuição geográfica: sul dos Estados Unidos da América, México, Colômbia, Peru, Brasil, Argentina, Uruguai (Lindquist 1982); Ilhas do Caribe, América do Sul (Jackson 1931a) Japão, Austrália (Hennen et al. 2005)

Puccinia psidii G. Winter, Hedwigia 23: 171. 1884. Bullaria psidii (G. Winter) Arthur \& Mains, N. Amer. Fl. 7: 488. 1922. TIPO: BRASIL. SANTA CATARINA: sobre Psidium guajava L. (registrado como Psidium pomiferum L.), IV-1884, Ule 14.

Figuras 15-16

Puccinia jambosae Henn., Hedwigia 41: 105. 1902. TIPO: BRASIL. São PaUlo: sobre Syzygium jambos (L.) Alston (registrado como Jambosa vulgaris DC.), 7-V-1901, Puttemans 223.

Puccinia cambucae Puttemans in Rangel, Arch. Mus. Nac. Rio de Janeiro 18: 153. 1916. TIPO: BRASIL. São PAUlo: sobre Marlierea edulis Nied., V-1911, Puttemans 411.

Puccinia brittoi Rangel, Arch. Mus. Nac. Rio de Janeiro 18: 154. 1916. TIPO: BRASIL. RIO DE JANEIRO: sobre Campomanesia maschalantha (O. Berg.) Kiaersk. (registrado como Abbevillea maschalantha O. Berg.), I-1914, E.S. Britto 1036.

Puccinia barbacensis Rangel, Arch. Mus. Nac. Rio de Janeiro 18: 154. 1916. TIPO: BRASIL. MinAS Gerais: Barbacena, sobre Myrtaceae indet. (Eugenia sp.?), III-1911, Puttemans 296.

Puccinia eugeniae Rangel, Arch. Mus. Nac. Rio de Janeiro 18: 154. 1916. TIPO: BRASIL. SÃo PAULO: sobre Eugenia grandis Wight, IV-1901, Rangel 261.
Puccinia grumixamae Rangel, Arch. Jard. Bot. Rio de Janeiro 2: 69. 1918. TIPO: BRASIL. RIO DE JANEIRO: sobre Eugenia brasiliensis Lam., s.d., Rangel 1515.

Puccinia jambolani Rangel, A Lavoura 16: 124. 1912. TIPO: BRASIL. Rio DE JANEIRo: Rio de Janeiro, sobre Syzygium jambolanum DC., VI-1912, Rangel s.n.

Puccinia camargoi Puttemans, Bol. Mus. Nac. Rio de Janeiro 6: 314. 1930. TIPO: BRASIL. São Paulo: Campinas, sobre Melaleuca leucodendra (L.) L., VII-1930, Camargo s.n.

Puccinia actinostemonis H.S. Jacks. \& Holw. in Jackson, Mycologia 23: 466. 1931. TIPO: BRASIL. SÃo PAulo: sobre Myrtaceae indet. (e não Actinostemon sp., Euphorbiaceae), 27-II-1922, Holway 1600.

Anamorfos

Uredo psidii J.A. Simpson, K. Thomas \& C.A. Grgurinovic, Aust. Plant Path. 35: 555. 2006. Caeoma eugeniarum Link in Linnaeus, Sp. Plant. 6: 29. 1825. Uredo eugeniarum (Link) Buriticá in Buriticá \& Pardo-Cardona, Rev. Acad. Colomb. Cienc. Exactas Fis. Nat. 20: 222. 1996. TIPO: BRASIL. RIO DE JANEIRO: sobre Eugenia sp., s.d., Beyrich s.n.

Uredo neurophila Speg., An. Soc. Cient. Argent. 17: 122. 1884. Puccinia neurophila (Speg.) Speg., Rev. Argent. Bot. 1: 120. 1925. TIPO: PARAGUAI. GUAIRÁ: Villa Rica, sobre Jambosa sp., I-1882, Balansa 3466.

Uredo flavidula Winter, Hedwigia 24: 260. 1885. TIPO: BRASIL. SANTA CATARINA: sobre Myrtaceae indet., s.d., Ule s.n.

Uredo myrtacearum Pazschke, in Rabenhorst \& Winter, Hedwigia 29: 159. 1890. TIPO: BRASIL. SANTA CARTARIna: sobre Eugenia sp., XII-1883, Ule 10.

Uredo eugeniarum Henn., Hedwigia 34: 337. 1895. LECTÓTIPO: BRASIL. SANTA CATARINA: Blumenau, sobre Eugenia sp., s.d., Moller 227.

Aecidium glaziovii Henn., Hedwigia 36: 216. 1897. TIPO: BRASIL. RIO DE JANEIRO: sobre Myrtaceae indet., s.d., Glaziou 20621.

Uredo pitanga Speg., An. Mus. Nac. Buenos Aires 6: 240. 1898. TIPO: URUGUAI. s.1., sobre Stenocalyx pitanga O. Berg., XII-1881, Arechavaleta s.n.

Uredo puttemansii Henn., Hedwigia 41:106. 1902. TIPO: BRASIL. São PAULO: sobre Myrtaceae, s.d., 


\section{Puttemans 197.}

Uredo goeldiana Henn., Hedwigia Beiblatt 42: 188. 1903. TIPO: BRASIL. PARÁ: Belém, sobre Eugenia sp., 1883, Goeldi s.n.

Uredo rochaei Puttemans, Rev. Polytechnica, São Paulo 11: 272. 1906. TIPO: BRASIL. São PAULO: sobre Myrciaria jaboticaba O. Berk, s.d., A. Rocha s.n.

Uredo myrciae Mayor, Mem. Soc. Neuchatel. Sci. Nat. 5: 590. 1913. LECTÓTIPO: COLÔMBIA. MEDELLín: sobre Myrcia cf. acuminata (Kunth) DC., 0, Mayor 209.

III - Teliossoros anfígenos, surgindo a partir dos urediniossoros, castanho-escuros, em áreas necróticas irregulares, pulverulentos, 0,5-1,2 mm diâm.; paráfises ausentes. Teliósporos misturados aos urediniósporos, elipsóides ou ovóides, fusiformes, arredondados no ápice, estreitos ou esféricos na base, ligeiramente constritos no septo, (35-)43(-51) $\mu \mathrm{m}$ compr., (14-)18(-22) $\mu \mathrm{m}$ larg.; parede castanho-claro, 0,7-1,5 $\mu \mathrm{m}$ espessa e uniforme; poro germinativo não visível; pedicelo decíduo, entretanto, pequena parte do mesmo permanece aderido à maioria dos teliósporos. II - Urediniossoros anfígenos, predominando os hipófilos, castanho-claros a amarelados, de origem subepidermal, algumas vezes tardiamente irrompentes, pulverulentos, ruptura da epiderme conspícua em manchas irregulares, ovóides ou oblongas, 0,5-1,2 mm diâm.; paráfises ausentes. Urediniósporos com pedicelos decíduos, elipsóides a obovóides, (19-)22(-24) $\mu \mathrm{m}$ compr., (13-)15(-17) $\mu \mathrm{m}$ larg.; parede hialina ou amarelo-clara, uniformemente equinulada, 1,2-2 $\mu$ m espessa lateral; poros germinativos obscuros. 0, I - Estádios espermogonial e ecial desconhecidos.

Materiais examinados: sobre Syzygium jambos (L.) Alston (Myrtaceae): 29-VII-1997, A.A. Carvalho Júnior 97-141 (IBI17911-II, III); 23-I-1998, 98-030 (IBI18046-II, III); 25-VII-1998, 98-345 (IBI18362II, III); sobre Psidium guajava L.: 24-X-1997, A.A. Carvalho Júnior 97-201 (IBI17971-II); 24-XI-1997, 97-260 (IBI17986-II); 22-XII-1997，97-236 (IBI18006-II); 13-I-1998, 98-011 (IBI18028-II); 20-II-1998, 98-036 (IBI18052-II); 31-III-1998, 98-274 (IBI18291-II, III); 23-IV-1998, 98-306 (IBI18323-II); 26-V-1998, 98-329 (IBI18346-II).

Comentários: segundo Hennen et al. (2005) Uredo subneurophila Speg. e Puccinia subneurophylla (Speg.) Speg. foram considerados por vários autores como sinônimos de Puccinia psidii.
No entanto, o espécime analisado, coletado por Balansa 3800 em julho de 1883, pertence a um gênero não identificado da família Apocynaceae, reportado originalmente como Psidium sp. (Myrtaceae). Ainda segundo o mesmo autor, o espécime coletado por Balansa tem soros com características não comuns para ferrugens, e trata-se de um gênero anamórfico ainda sem nome sobre Apocynaceae. Alguns autores como Averna-Saccá (1920), Arthur (1922) e Hennen et al. (2005) citam como protólogo da sinonímia teleomórfica Puccinia jambolani Rangel. Entretanto, a citação correta e a que adotamos neste trabalho é da publicação de Rangel (1912), na qual se encontra a descrição em Latim.

Existe certa confusão na literatura sobre o autor de Caeoma eugeniarum. Lindquist (1982) considera como sendo Winter. Sydow \& Sydow (1924) e Hennen et al. (2005) consideram como sendo Linnaeus; no entanto, Hennen et al. (2005) comentam que não tiveram acesso à publicação. Buriticá \& PardoCardona (1996) atribuem a Link. Após análise das publicações concluímos que a citação completa e correta da espécie é Caeoma eugeniarum Link, descrita na obra Species Plantarum. Buriticá \& PardoCardona (1996) sugeriram Uredo eugeniarum (Link) Buriticá como combinação anamórfica nova de C. eugeniarum Link; entretanto, esta combinação constitui um homônimo posterior à Uredo eugeniarum Henn. e, segundo Greuter et al. (2000), deve ser rejeitado. Mais recentemente, Simpson et al. (2006) sugeriram Uredo psidii J.A. Simpson, K. Thomas \& C.A. Grgurinovic como novo nome para C. eugeniarum Link, que segundo nosso entendimento, deve ser adotado.

Distribuição geográfica: Colômbia, Brasil, Paraguai, Argentina, Uruguai (Lindquist 1982, PardoCardona 1994).

Sphenospora smilacina Syd., Ann. Mycol. 23: 318. 1925. TIPO: GUATEMALA. Retalhuleu: São Felipe, sobre Smilax sp., 14-I-1917, Holway 718. Figuras 17-18

Anamorfos

Uredo yurimaguasensis Henn., Hedwigia 43: 164.

1904. Sphenospora yurimaguasensis H.S. Jacks. \& Holw. in Jackson, Mycologia 18: 153. 1926. TIPO: PERU. LORETO: Yurimaguas, sobre Smilax sp., VIII-1902, Ule 3251.

III - Teliossoros hipófilos, avermelhados a amarelomel, muito cerosos, irrompentes, expostos desde cedo, 

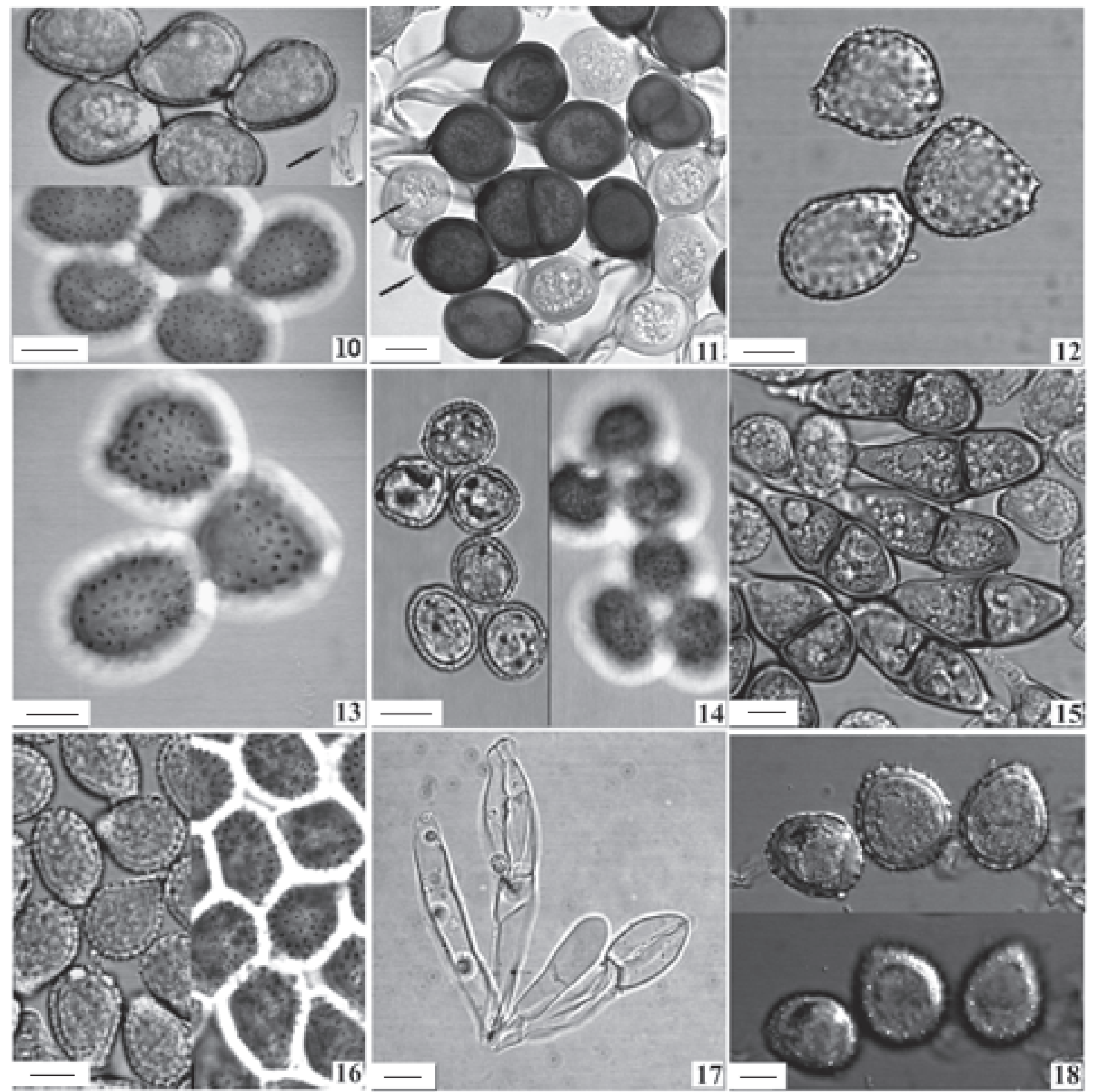

Figura 10. Phakopsora colubrina (IBI18369). 10. Acima, urediniósporos em vista mediana e à direita (seta), aspecto da paráfise do soro uredinial. 10. Abaixo, urediniósporos em vista superficial. Figura 11. Teliósporos de Puccinia arechavaletae (IBI17966) com setas indicando teliósporos unicelulares (mesósporos). Figuras 12-13. Urediniósporos de Puccinia lygodii (IBI18301). 12. Em vista mediana. 13. Em vista superficial. Figura 14. Puccinia oxalidis (IBI17987). 14. À esquerda, urediniósporos em vista mediana e à direita, em vista superficial. Figuras 15-16. Puccinia psidii (IBI18291). 15. Teliósporos (e alguns urediniósporos). 16. Urediniósporos, à esquerda em vista mediana e à direita em vista superficial. Figuras 17-18. Sphenospora smilacina (IBI18041). 17. Teliósporos. 18. Urediniósporos, acima em vista mediana e abaixo em vista superficial. Barras $=10 \mu \mathrm{m}$. 
agrupados desordenadamente ou em círculos concêntricos, também circundando soros urediniais, 0,1-0,5 mm diâm.; paráfises ausentes. Teliósporos oblongos, fusiformes, arredondados acima e abaixo, (19-)23(-25) $\mu \mathrm{m}$ compr., (10-)12(-14) $\mu \mathrm{m}$ larg.; parede quase hialina ou hialina, de espessura uniformemente fina, lisa; poros germinativos não visíveis; pedicelos hialinos, 15-35 $\mu \mathrm{m}$ compr.

II - Urediniossoros geralmente hipófilos, em regiões cloróticas, amarelo-ouro a canela-claros, dispersos, agrupados ou em círculos concêntricos, desde cedo expostos, também por uma ruptura longitudinal, muito pulvinado, rompendo a epiderme conspicuamente, arredondado-irregular ou alongado, 0,2-0,5 mm diâm.; paráfises ausentes. Urediniósporos com pedicelos

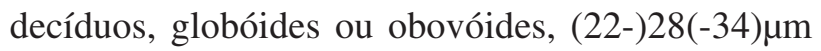
compr., (17-)22(-27) $\mu \mathrm{m}$ larg.; parede de coloração canela, densamente equinulada, 1,2-3 $\mu$ m espessa; 2 poros germinativos aproximadamente equatoriais.

0, I - Estádios espermogonial e ecial são desconhecidos.

Materiais examinados: sobre Smilax quinquenervia Vell. (Smilacaceae): 13-I-1998, A.A. Carvalho Júnior 98-009 (IBI18026-II); 23-I-1998, 98-024 (IBI18041, RB382837-II, III); 20-II-1998, 98-039 (IBI18055-II); 31-III-1998, 98-276 (IBI18293-II); 23-IV-1998, 98299 (IBI18316-II); 25-VII-1998, 98-350 (IBI18367-II).

Comentários: a denominação da espécie publicada por Jackson (1926) é nula pois, além do autor não ter feito uma descrição da nova combinação, em 1925 já havia sido publicada por Sydow (1925) a descrição da espécie em Latim.

Distribuição geográfica: México, América Central, Ilhas do Caribe, Venezuela, Colômbia, Equador, Peru e Brasil (Jackson 1926, Hennen et al. 2005).

\section{Agradecimentos}

Os autores agradecem ao CNPq, à FAPERJ e à FAPESP pelo apoio financeiro; ao Dr. Mílton Groppo Júnior, pelo auxílio na identificação das espécies hospedeiras e ao Sr. Craig Brough do RBG-Kew pelo auxílio na referência bibliográfica de Caeoma eugeniarum

\section{Literatura citada}

Arthur, J.C. 1962. Manual of the rusts in United States and Canada. Hafner Publishing, New York.
Arthur, J.C. 1920. Uredinales - Aecidiaceae (continuatio). North American Flora 7: 337-404.

Arthur, J.C. 1922. Uredinales - Aecidiaceae (continuatio). North American Flora 7: 481-540.

Averna-Sacca, R. 1920. Moléstias do cambucazeiro (Rubachia glomerata). Boletim de Agricultura (São Paulo) 21:37-42.

Buriticá, P.C. 1999. La familia Phakopsoraceae en el Neotrópico III, Géneros: Batistopsora y Phakopsora. Revista de la Academia Colombiana de Ciências Exactas, Físicas y Naturales 23: 271-305.

Buriticá, P. \& Hennen, J.F. 1980. Pucciniosireae (Uredinales, Pucciniaceae). Flora Neotropica 24: $1-50$.

Buriticá, P. \& Pardo-Cardona, V.M. 1996. Flora Uredineana Colombiana. Revista de la Academia Colombiana de Ciências Exactas, Físicas y Naturales 20: 183-236.

Carvalho Júnior, A.A. 2001. Micota Uredinológica da Reserva Florestal "Armando de Salles Oliveira" (São Paulo, Brasil). Tese de Doutorado, Universidade Estadual Paulista, Botucatu.

Carvalho Júnior, A.A., Figueiredo, M.B., Furtado, E.L. \& Hennen, J.F. 2002a. Micota Uredinológica da Reserva Florestal "Armando de Salles Oliveira", São Paulo, Brasil: sobre Amaranthaceae, Asclepiadaceae, Bignoniaceae e Boraginaceae. Hoehnea 29: 19-30.

Carvalho Júnior, A.A., Figueiredo, M.B., Furtado, E.L. \& Hennen, J.F. 2002b. Uredinales sobre Asteraceae da Reserva Florestal "Armando de Salles Oliveira", São Paulo, Brasil. Hoehnea 29: 57-64.

Carvalho Júnior, A.A., Figueiredo, M.B., Furtado, E.L. \& Hennen, J.F. 2004a. Uredinales sobre Poaceae da Reserva Florestal "Armando de Salles Oliveira", São Paulo, SP, Brasil. Hoehnea 31: 313-320.

Carvalho Júnior, A.A., Figueiredo, M.B. \& Hennen, J.F. 2004b. Catálogo dos fungos causadores de ferrugens (Uredinales) do Parque Estadual das Fontes do Ipiranga, São Paulo, SP. Hoehnea 31: 51-60.

Carvalho Júnior, A.A., Figueiredo, M.B., Furtado, E.L. \& Hennen, J.F. 2006. Uredinales sobre Convolvulaceae, Cucurbitaceae, Euphorbiaceae, Fabaceae, Malvaceae, Marantaceae e Moraceae da Reserva Florestal “Armando de Salles Oliveira", São Paulo, SP, Brasil. Hoehnea 33: 317-329.

Greuter, W., McNeill, J., Barrie, F.R., Burdet, H.M, Demoulin, V., Filgueiras, T.S, Nicolson, D.H., Silva, P.C., Skog, J.E., Trehane, P., Turland, N.J. \& Hawksworth, D.L. 2000. International Code of Botanical Nomenclature (Saint Louis Code). Regnum Vegetabile 138: $1-474$.

Cummins, G.B. \& Hiratsuka, Y. 2003. Illustrated genera of rust fungi. 3 ed. A.P.S. Press, Saint Paul.

Gallegos, H.L. \& Cummins, G.B. 1981a. Uredinales (Royas) de México. v.1. SARH, Culiacán. 
Gallegos, H.L. \& Cummins, G.B. 1981b. Uredinales (Royas) de México. v. 2. SARH, Culiacán.

Hennen, J.F. \& Figueiredo, M.B. 1984. The life cycle of Hemileia vastatrix? In: Comunicações do Simpósio sobre ferrugens do cafeeiro, 1983, Oeiras. Oeiras: Centro de Investigação da Ferrugem do Cafeeiro, pp. 47-56.

Hennen, J.F, Figueiredo, M.B, Carvalho Júnior, A.A. \& Hennen, P.G. 2005. Catalogue of species of plant rust fungi (Uredinales) of Brazil. http:// www.jbrj.gov.br/ (acesso em 1.11.2006).

Jackson, H.S. 1926. The rusts of South America based on the Holway Collections-I. Mycologia 18: 139-162.

Jackson, H.S. 1931a. The rusts of South America based on the Holway Collections-IV. Mycologia 23: 332364.

Jackson, H.S. 1931b. The rusts of South America based on the Holway Collections-V. Mycologia 23: 463-503.

Lindquist, J.C. 1982. Royas de la republica Argentina y zonas limitrofes. Instituto Nacional de Tecnología Agropecuária, Buenos Aires.

Long, W.H. \& Harsch, R.M. 1918. Aecial stage of Puccinia oxalidis. Botanical Gazette 65: 475-477.

Olive, E.W. \& Whetzel, H.H. 1917. Endophyllum-like rusts of Porto Rico. American Journal of Botany 4: 44-52.

Pardo-Cardona, V.M. 1994. Indice comentado de las royas (Fungi, Uredinales) del departamento de Antioquia,
Colombia, S.A. Revista del Instituto de Ciências Naturales y Ecologia 5: 99-172.

Rajendren, R.B. 1967. Atypical and typical germination of uredinoid teliospores of Hemineia vastatrix. Mycologia 59: 918-921.

Rangel, E. 1912. Nova moléstia do Jamelão (Syzygium jambolanum DC). A Lavoura 16: 123-124.

Rodrigues Júnior, C.J., Rijo, L. \& Medeiros, E.F. 1980. Germinação anômala dos uredósporos de Hemileia vastatrix, o agente causal da ferrugem alaranjada do cafeeiro. Garcia de Orta, Série de Estudos Agronômicos 7: 17-20.

Simpson, J.A., Thomas, K. \& Grgurinovic, C.A. 2006. Uredinales species pathogenic on species of Myrtaceae. Australasian Plant Pathology 35: 549-562.

Sotão, H.M.P., Hennen, J.F. \& Cavalcante, M.A. 2001. Uredinales do Estado do Amapá: Gênero Puccinia. Boletim do Museu Paraense Emílio Goeldi, série Botânica 17: 107-159.

Sydow, H. 1925. Fungi in itinere costarricensis colecti, pars prima. Annales Mycologici 23: 308-429.

Sydow, P. \& Sydow, H. 1924. Monographia uredinearum specierum omnium ad hunc usque diem cognitarum descriptio et adumbratio systematica IV: Uredineae Imperfecti (Peridermium, Aecidium, Monosporidium, Roestelia, Caeoma, Uredo, Mapea). Fratres Borntraeger, Leipzig. 